\title{
MELD score and AST-to-platelet ratio index predict long-term survival in patients with a small hepatocellular carcinoma following non-transplant therapies: a pilot study
}

\author{
Joy Sarkar ${ }^{1}$, Thomas DeLeon², Linda L. Wong ${ }^{3}$ \\ ${ }^{\prime}$ Tripler Army Medical Center, Surgery, Honolulu, HI 96813, USA. \\ ${ }^{2}$ Department of Medicine, John A. Burns School of Medicine, Honolulu, HI 96813,USA. \\ ${ }^{3}$ Department of Surgery, John A. Burns School of Medicine, Honolulu, HI 96813, USA.
}

Correspondence to: Prof. Linda L. Wong, Department of Surgery, John A. Burns School of Medicine, 550 S. Beretania Street, Suite 403, Honolulu, HI 96813, USA. E-mail: Hepatoma@aol.com

How to cite this article: Sarkar J, DeLeon T, Wong LL. MELD score and AST-to-platelet ratio index predict long-term survival in patients with a small hepatocellular carcinoma following non-transplant therapies: a pilot study. Hepatoma Res 2017;3:79-85.

Article history:

Received: 26-02-2017

Accepted: 13-04-2017

Published: 09-05-2017

Key words:

Hepatocellular carcinoma,

ablation,

liver resection,

transplant

\begin{abstract}
Aim: Liver transplantation (LT) is the most effective treatment for long-term survival from hepatocellular carcinoma (HCC); however, insufficient donors limit therapy. The authors sought to identify characteristics that predicted long-term survival after non-transplant therapies in patients with small HCC. Methods: In a database of 1,050 HCC patients, the authors identified those with single $\mathrm{HCC} \leq 3.0 \mathrm{~cm}$, who underwent hepatic resection (HR, $n$ $=16$ ), radiofrequency ablation (RFA, $n=55)$, or LT $(n=23)$ with 5 -year follow-up. Overall survival (OS) and odds-ratios (OR) for survival after HR/RFA were calculated for MELD score, platelet count, creatinine, albumin, AST/platelet ratio index (APRI), international normalized ratio, and bilirubin. Results: LT patients had 3- and 5-year OS of $82.6 \%$ and $73.9 \%$ compared to HR/RFA patients with 3- and 5-year OS of $40.8 \%$ and $33.8 \%$. The strongest predictors of survival after HR/RFA were MELD $<10$ [OR 4.43, 95\% confidence interval (CI) 1.85-10.58] and APRI $\leq 0.5$ (OR 4.25, 95\% CI 1.63-11.08). HR/RFA patients with both MELD $<10$ and APRI $\leq 0.5$ had 3- and 5-year OS of $77.3 \%$ and $72.7 \%$. Conclusion: Patients with MELD $<10$ and APRI $\leq 0.5$ who undergo HR/RFA have survival approaching LT. Perhaps patients who meet these criteria can safely undergo non-transplant therapy and donor livers can be allocated to patients with a greater need.
\end{abstract}

\section{INTRODUCTION}

Hepatocellular carcinoma (HCC) is the most common primary malignancy of the liver. Worldwide, there were 782,000 new cases in 2012 and HCC is the second leading cause of cancer-related mortality with 745,000 deaths. ${ }^{[1,2]}$ Advanced stage at diagnosis and poor underlying liver function present major challenges to treatment. Potential curative therapies for HCC include hepatic resection (HR) and liver 
transplantation (LT). LT is viewed as the optimal treatment for HCC as it treats both the tumor and the underlying liver disease ${ }^{[3]}$ However, the inadequate number of available donors significantly limits use of LT. Prolonged waiting times lead to dropout from the waiting list due to tumor progression exceeding criteria for LT, or death due to liver failure. ${ }^{[4]}$ While overall survival (OS) and recurrence-free survival are both higher in patients undergoing LT compared to $H R$, prior studies have found that resection in patients with a single tumor less than $3.0 \mathrm{~cm}$ in size may have comparable survival to those undergoing $L T .{ }^{[5]}$ Similarly, radiofrequency ablation (RFA), while not a curative therapy, is a safe and effective alternative to $H R$ in patients who are not surgical candidates. Direct comparisons of overall survival between HR and RFA are limited by the degree of hepatic dysfunction in the patients who are offered resection versus ablation, but retrospective studies suggest that survival after RFA may not differ significantly from that of HR in certain patient populations. ${ }^{[6,7]}$

Prognosis is also affected by the degree of hepatic dysfunction, patient comorbidities, and tumor biology. Increasing evidence suggests that tumor size is a surrogate marker of tumor biology and surgical outcomes. Tumors less than $3.0 \mathrm{~cm}$ have been shown to be well-differentiated, contained within the capsule and have better prognosis. ${ }^{[8]}$ Smaller tumors have a higher likelihood of being successfully treated by nontransplant therapies. Therefore, our goal is to identify characteristics in patients with small $\mathrm{HCC}(\leq 3.0 \mathrm{~cm})$ that predict comparable long-term survival after HR or RFA versus $L T$, as these patients may be able to undergo non-transplant therapy and allow allocation of donor livers to those most in need.

\section{METHODS}

\section{Patients}

This is a retrospective analysis of 94 patients out of a cohort of $1,050 \mathrm{HCC}$ cases referred over a 22year period (1993-2014) to our group of physicians associated with the only liver transplant program in Hawaii, and the only referral center for liver disease/ surgery for American territories of the Pacific Basin (including Samoa, Guam, Saipan, and the Marshall Islands). Patients also included foreign nationals from China, Japan, Korea, and the Philippines, who sought medical care in the USA. About $75 \%$ of the overall cohort had some type of viral hepatitis with about $41 \%$ with hepatitis C, $38 \%$ hepatitis B and $4-5 \%$ coinfected with both. This center sees about $65-70 \%$ of the HCC cases in Hawaii. This study was approved by the University of Hawaii Institutional Review Board.

\section{HCC diagnosis}

Patients with either a histological or clinical diagnosis of HCC were considered for inclusion. Histological diagnosis of HCC was made either from liver biopsy or examination of the resected liver. Patients without histologic diagnosis, but a history of chronic liver disease, mass $>2 \mathrm{~cm}$ in size on dynamic imaging and one of the following (1) arterial uptake with venous washout seen on computed tomography scan or magnetic resonance imaging or (2) alpha-feto protein $($ AFP) $>200 \mathrm{ng} / \mathrm{mL}$.

\section{Study design}

Inclusion criteria were the following: (1) patients with a single tumor $\leq 3.0 \mathrm{~cm}$; (2) treatment with HR, RFA, or LT; and (3) either minimum follow-up of at least 5 years or death prior to the 5-year mark. We excluded 865 patients with multiple tumors or tumors $\geq 3.0 \mathrm{~cm}$. Of the remaining 185 patients, 69 were lost to followup prior to the 5 -year mark or were enrolled less than 5 years prior to the time of data analysis and 22 received another therapy (chemoembolization, Yttrium-90 or sorafenib) or no therapy. The final study population included 94 patients: 55 patients underwent RFA as their sole therapy, 16 underwent HR and 23 had LT.

Demographic/medical data were collected prospectively via clinical interview and chart analysis, and the data retrospectively analyzed. Patient characteristics chosen for analysis were: age $\leq 50$ years, age $\leq 60$ years, gender, presence of hepatitis $\mathrm{B}$ and/or hepatitis $\mathrm{C}$, alcohol use (defined as 2 or more alcoholic drinks/day for 10 years), obesity [defined as body mass index (BMI) $\geq 30$ ], smoking, diabetes mellitus, AFP (stratified as normal versus abnormal with normal $<20 \mathrm{ng} / \mathrm{dL}$ ), tumor size $\leq 1.5 \mathrm{~cm}$, presence of cirrhosis, serum bilirubin $\leq 1.2 \mathrm{mg} / \mathrm{dL}$, albumin $\geq$ $2.5 \mathrm{~g} / \mathrm{dL}$, albumin $\geq 3.0 \mathrm{~g} / \mathrm{dL}$, international normalized ratio $(I N R) \leq 1.2, I N R \leq 1.7$, presence of ascites, Child-Turcotte-Pugh (CTP) score $\leq \mathrm{A}$, CTP score $\leq \mathrm{B}$, platelet count $\geq 100$, creatinine $\leq 1.0 \mathrm{mg} / \mathrm{dL}$, AST-toplatelet ratio index (APRI) $\leq 0.5, A P R I \leq 1$, and MELD score $<10$. Laboratory data used for the study was obtained within 2 weeks of the initial visit. Exception points were added to the MELD scores of patients with $\mathrm{HCC}$ whose tumors met Milan criteria, in order to balance their risk of tumor progression and dropout to that of non-HCC patients. Because the number of added exception points fluctuated throughout the study period based on united network for organ sharing guidelines, the raw MELD score rather than the adjusted MELD score was used in the analysis for consistency. APRI was categorized based on initial description by Wai et al ${ }^{[9]}$ Of patients with an APRI of $\leq 0.5,85 \%$ would not have significant fibrosis (defined 
Table 1: Characteristics of study population by group

\begin{tabular}{|c|c|c|c|c|c|}
\hline Characteristic & $\mathrm{HR}(n=16)$ & RFA $(n=55)$ & $\mathrm{LT}(n=23)$ & Total $(n=94)$ & $P$ value \\
\hline Age (years), mean \pm SD & $58.6 \pm 8.3$ & $65.4 \pm 10.8$ & $54.3 \pm 6.2$ & $62.0 \pm 11.0$ & $<0.001^{*}, \dagger$ \\
\hline Male, $n(\%)$ & $13(81.3 \%)$ & $37(67.3 \%)$ & $21(91.3 \%)$ & $71(75.5 \%)$ & 0.067 \\
\hline Cirrhosis, $n(\%)$ & $12(75 \%)$ & $51(91.1 \%)$ & $23(100.0 \%)$ & $86(91.5 \%)$ & $0.020^{\ddagger}$ \\
\hline $\mathrm{HBV}, n(\%)$ & $6(37.5 \%)$ & $15(27.3 \%)$ & $8(34.8 \%)$ & $29(30.9 \%)$ & 0.661 \\
\hline $\mathrm{HCV}, n(\%)$ & $7(43.8 \%)$ & $31(56.4 \%)$ & $14(60.1 \%)$ & $52(55.3 \%)$ & 0.555 \\
\hline Diabetic, $n(\%)$ & $1(6.3 \%)$ & $16(29.1 \%)$ & $4(17.4 \%)$ & $21(22.3 \%)$ & 0.125 \\
\hline $\mathrm{BMI}$, mean $\pm \mathrm{SD}$ & $22.7 \pm 3.3$ & $25.4 \pm 5.5$ & $29.4 \pm 4.9$ & $26.0 \pm 6.0$ & $<0.001^{\ddagger, \dagger}$ \\
\hline Ascites, $n(\%)$ & $0(0.0 \%)$ & $18(32.7 \%)$ & $8(34.8 \%)$ & $26(27.7 \%)$ & $0.025^{\star, \neq}$ \\
\hline Serum bilirubin $(\mathrm{mg} / \mathrm{dL})$, mean $\pm \mathrm{SD}$ & $1.1 \pm 0.7$ & $1.5 \pm 1.0$ & $1.8 \pm 1.1$ & $1.5 \pm 1.0$ & 0.097 \\
\hline MELD score, mean \pm SD & $8 \pm 2$ & $11 \pm 4$ & $11 \pm 4$ & $11 \pm 4$ & $0.016^{*}, \neq$ \\
\hline Platelet count $\left(\times 10^{3} / \mu \mathrm{L}\right)$, mean \pm SD & $139 \pm 46$ & $119 \pm 69$ & $109 \pm 76$ & $121 \pm 69$ & 0.394 \\
\hline Tumor size $(\mathrm{cm})$, mean \pm SD & $2.0 \pm 0.6$ & $2.3 \pm 0.5$ & $2.3 \pm 0.5$ & $2.3 \pm 0.5$ & 0.114 \\
\hline
\end{tabular}

${ }^{\star} P<0.05$, HR vs. RFA; ${ }^{\dagger} P<0.05$, RFA vs. LT; ${ }^{\ddagger} P<0.05$, HR vs. LT. HR: hepatic resection; RFA: radiofrequency ablation; LT: liver transplantation; HBV: hepatitis B virus; HCV: hepatitis C virus; BMI: body mass index

as an Ishak score of 3 or more), and of patients with an APRI of $\leq 1.00,98 \%$ would not have cirrhosis (defined as an Ishak score of 5 or 6 ). Thus we chose the cutoff values of APRI $\leq 0.5$ and $\leq 1.00$ for our study. Outcome measures included: 3- and 5-year survival and recurrence categorized as early $(<2$ years) vs. late ( $\geq 2$ years).

\section{Statistical analysis}

One-way analysis of variance was used to identify significant differences between the baseline characteristics of the three study groups defined by continuous variables: age, BMI, serum bilirubin, MELD score, platelet count, and tumor size. For groups in which a difference was identified, the Tukey post-hoc analysis was applied to determine which of the three comparisons (HR vs. RFA, HR vs. LT, RFA vs. LT) contained the difference. The chi-squared test was used to identify significant differences between the baseline characteristics of the 3 groups defined by categorical variables: gender, presence of cirrhosis, presence of hepatitis B or C, presence of diabetes, and presence of ascites. For groups in which a difference was identified, the Fisher's exact test was used to determine which of the 3 comparisons contained the difference.

Odds ratios (OR) were calculated for both 3-year and 5 -year OS for each of the patient characteristics, in each of the groups HR, RFA, LT. The OS for HR and RFA groups were calculated both separately and as a composite (HR/RFA), and compared against OS for patients undergoing LT. Results were expressed as OR with $95 \%$ confidence interval $(\mathrm{Cl})$. Only results with a $P$ value $<0.05$ were considered statistically significant.

\section{RESULTS}

\section{Baseline characteristics}

The demographics of the patients included in this study are outlined in Table 1. The patients were 71 men and 23 women with a mean age of $62 \pm 11$ years and $1 \mathrm{HCC}$ tumor with a mean size of $2.3 \pm 0.5 \mathrm{~cm}$. The majority of patients $(73.4 \%)$ were Asian or Pacific Islander. The RFA group differed significantly from the HR and LT groups with respect to age (mean age of 65.4 vs. 58.6 and 54.3 years respectively). The HR group differed significantly from the RFA and LT groups with respect to presence of ascites and MELD score: $0 \%$ with ascites in HR group vs. $32.7 \%$ and $34.8 \%$, respectively. The mean MELD score in the HR group was 8, compared to 11 in the RFA and LT groups. Patients in the LT group had a significantly higher BMI than patients in the HR and RFA groups (29.4 vs. 22.7 and 25.4, respectively). Finally, the HR and LT groups differed significantly with respect to cirrhosis: $75 \%$ in the HR vs. $100 \%$ of LT patients.

Of the 23 patients who underwent LT, 15 underwent locoregional therapy before LT including 2 patients who underwent resection, 8 RFA and 6 transcatheter arterial chemoembolization (TACE) procedures; 2 patients received both RFA and TACE while awaiting transplant. Mean waiting time for LT was 355 days (range 120-720 days). Mean MELD score was similar between the LT and RFA groups. Most patients who underwent $L T$ received MELD exception points in order to qualify for transplant as only 3 patients had MELD above 15.

\section{Overall survival}

Overall 3-year and 5-year survival in all patients undergoing $L T$ was significantly higher than patients in the HR, RFA, and composite HR/RFA groups [Table 2]. The 3-year survival was $82.6 \%$ in the LT group, $62.5 \%$ in the HR group, $34.5 \%$ in the RFA group, and $40.8 \%$ in the composite HR/RFA group. Similarly, 5-year survival rates were $73.9 \%, 56.3 \%, 27.3 \%$, and $33.8 \%$ respectively. 
Table 2: The 3-year and 5-year OS after LT, HR, and RFA

\begin{tabular}{lcccc}
\hline Survival & LT & HR & RFA & HR/RFA \\
\hline 3-year OS & $82.6 \%$ & $62.5 \%$ & $34.5 \%$ & $40.8 \%$ \\
5-year OS & $73.9 \%$ & $56.3 \%$ & $27.3 \%$ & $33.8 \%$ \\
\hline
\end{tabular}

LT: liver transplantation; HR: hepatic resection; RFA: radiofrequency ablation; OS: overall survival

\section{Patient characteristics significantly affecting survival}

Patient characteristics with statistically significant ORs for both 3-year and 5-year OS were: MELD < 10 , creatinine $\leq 1 \mathrm{mg} / \mathrm{dL}$, and APRI $\leq 0.5$ [Table 3]. Characteristics with significant ORs inversely correlating with 3-year and 5-year OS were age $>60$ years and presence of diabetes. Serum bilirubin $\leq 1.2 \mathrm{mg} / \mathrm{dL}$, serum albumin $\geq 3.0 \mathrm{~g} / \mathrm{dL}$, and CTP score $\leq 6$ approached but did not reach significance.

\section{Modified OS}

Modified 3-year and 5-year OS was calculated for patients who underwent HR or RFA with the characteristics in Table 3, and compared with 3-year and 5-year survival after LT [Figure 1]. APRI $\leq 0.5$ was associated with a 3-year OS of $68.0 \%$ and 5 -year OS of $64.0 \%$, and MELD < 10 was associated with a 3 -year OS of $64.9 \%$ and 5 -year OS of $54.1 \%$. Patients who underwent HR or RFA with both MELD < 10 and APRI $\leq 0.5$ (22 out of 71 patients) had a modified 3 -year OS of $77.3 \%$ and 5 -year OS of $72.7 \%$. Diabetes mellitus was associated with a 3-year and 5-year OS of $17.6 \%$ following HR/RFA.

\section{Recurrence}

Of the 71 patients that underwent HR/RFA, 31 patients had documented recurrence, or $43.6 \%$. Twenty-seven of these 31 patients underwent subsequent treatment including 1 patient who underwent repeat resection, 16 patients who underwent RFA, 9 who underwent
TACE, and 6 patients who received chemotherapy (5 patients received more than 1 treatment modality for recurrence). Forty-four patients (including 3 out of the 31 patients with recurrence) did not have any documented subsequent therapy, so their causes of death were unclear. Among the 22 patients who underwent HR/RFA and had both MELD $<10$ and $\mathrm{APRI} \leq 0.5,13$ patients had a documented recurrence $(59.1 \%)$. In the remaining 49 HR/RFA patients, 18 patients had a documented recurrence (36.7\%). Two of the 23 patients who underwent LT had recurrence $(8.7 \%): 1$ patient had a local recurrence which was treated with RFA and sorafenib, and 1 patient underwent excision of a metastatic lesion on the chest wall.

The average time to recurrence among all HR/RFA patients was 935 days. Among the 22 patients with MELD $<10$ and APRI $\leq 0.5,5$ patients had early recurrence $(38.4 \%)$, and mean time to recurrence was 1,107 days (range 169-3,380 days). For the other $49 \mathrm{HR} / \mathrm{RFA}$ patients, 1 patient had recurrence for which time to recurrence was unknown, and 11 patients $(64.7 \%)$ had early recurrences. The average time to recurrence in this group was 803 days (range 188-2,664 days). There was a trend toward late recurrences in the low MELD/APRI group compared to the other patients $(61.5 \%$ vs. $35.3 \%)$, however this was not statistically significant $(P=0.27)$.

\section{DISCUSSION}

Determining the most appropriate initial therapy for early HCC is challenging given the need to balance procedural morbidity and mortality with long-term recurrence rates. LT has been shown in multiple retrospective studies and a meta-analysis to have superior long term, recurrence-free survival compared to $\mathrm{HR} \cdot{ }^{[10-14]}$ However, the scarcity of donor livers is a

Table 3: Patient characteristics significantly affecting 3-year and 5-year OS

\begin{tabular}{|c|c|c|c|c|c|}
\hline Characteristic & $n$ & $\begin{array}{c}\text { 3-year OS } \\
\text { OR }(95 \% \mathrm{Cl})\end{array}$ & $P$ value & $\begin{array}{l}\text { 5-year OS } \\
\text { OR (95\% Cl) }\end{array}$ & $P$ value \\
\hline MELD $<10$ & 54 & $4.43(1.85,10.58)$ & 0.0008 & $2.77(1.19,6.46)$ & 0.0181 \\
\hline APRI $\leq 0.5$ & 31 & $4.25(1.63,11.08)$ & 0.0031 & $4.09(1.59,10.50)$ & 0.0034 \\
\hline Creatinine $\leq 1.0 \mathrm{mg} / \mathrm{dL}$ & 55 & $6.28(2.09,18.86)$ & 0.0010 & $4.15(1.39,12.36)$ & 0.0107 \\
\hline Diabetes & 22 & $0.22(0.07,0.66)$ & 0.0070 & $0.32(0.11,0.97)$ & 0.0438 \\
\hline Age $>60$ years & 41 & $0.42(0.18,0.96)$ & 0.0396 & $0.32(0.14,0.75)$ & 0.0089 \\
\hline Bilirubin $\leq 1.2 \mathrm{mg} / \mathrm{dL}$ & 46 & $2.28(0.99,5.24)$ & 0.0520 & $2.19(0.95,5.06)$ & 0.0677 \\
\hline Albumin $\geq 3.0 \mathrm{~g} / \mathrm{dL}$ & 67 & $3.50(1.22,10.07)$ & 0.0201 & $2.36(0.82,6.78)$ & 0.1093 \\
\hline CTP score $\leq 6$ & 58 & $2.23(0.95,5.21)$ & 0.0649 & $2.00(0.84,4.74)$ & 0.1155 \\
\hline
\end{tabular}

OS: overall survival; OR: odds ratio; Cl: confidence interval; MELD: Model for End Stage Liver Disease; APRI: AST-to-platelet ratio index; CTP: Child-Turcotte-Pugh 


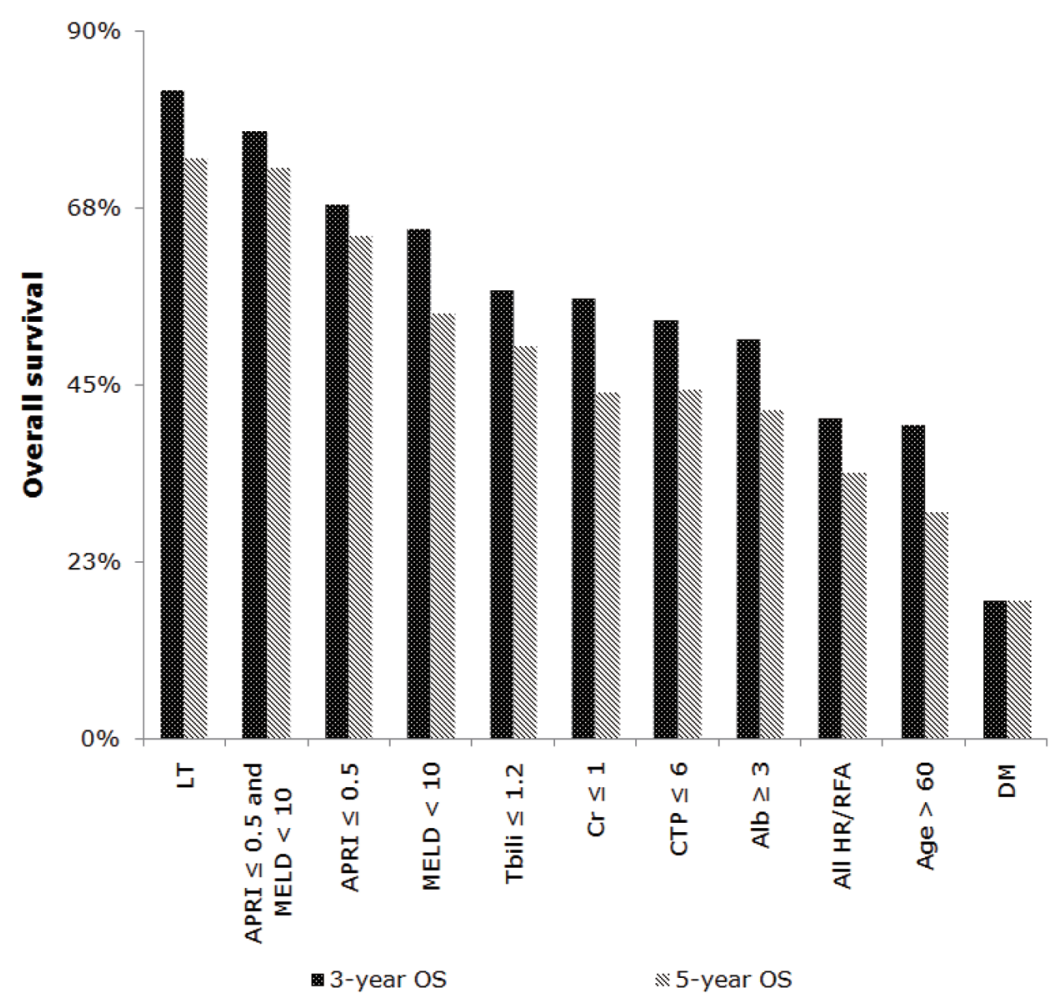

Figure 1: Modified overall 3-year and 5-year survival for selected patient characteristics. LT: liver transplantation; APRI: AST-to-platelet ratio index; MELD: Model for End Stage Liver Disease; Tbili: total bilirubin; Cr: creatinine; CTP: Child-Turcotte-Pugh score; Alb: albumin; HR: hepatic resection; RFA: radiofrequency ablation; DM: diabetes mellitus

limiting factor to transplantation in patients who meet criteria. Prolonged waiting times may lead to tumor progression and/or death from liver failure, and the estimated monthly drop-out rate increases with length of time on the waitlist, reaching $5.6 \%$ at 12 months. ${ }^{[4]}$ Because of limited donors, resection has been recommended for those with better liver function. ${ }^{[13,15]}$

Perhaps the biggest dilemma is how to treat the very small $\mathrm{HCC}$, especially those that do not meet minimum transplant criteria. Previous studies have shown good short-term outcome for small HCC whether ablated or resected, however recurrences are more frequent with RFA. ${ }^{[16]}$ Liu et al. ${ }^{[17]}$ in 237 patients with single $\mathrm{HCC}<2.0 \mathrm{~cm}$, concluded that resection provided better overall and recurrence-free survival than RFA and they recommended resection as the first line therapy. Other approaches have included the "wait and not ablate" tactic in small tumors - allowing tumors to progress until patients qualified for liver transplant. ${ }^{[18,19]}$

Some patients do have long-term survival after HR or RFA for $\mathrm{HCC}<3.0 \mathrm{~cm}$, but few studies identify factors that are predictive of a good outcome in the absence of transplantation. In this study, we demonstrate that although the overall 3-year and 5-year survival rates vary drastically between the HR and RFA groups
(62.5\% vs. 34.5\% 3-year OS, 56.3\% vs. 27.3\% 5-year OS), hepatic function is also very different. No patient in the HR group had ascites, vs. $33 \%$ of RFA patients, and $75 \%$ of HR patients were cirrhotic compared to $91 \%$ of RFA patients. We found that MELD $<10$, APRI $\leq 0.5$ and creatinine $<1.0$ were the best factors that predicted survival. Most importantly, when patients had both MELD < 10 and APRI $\leq 0.5$ and underwent HR or RFA, the 3- and 5-year survival was similar to those that underwent LT-despite the higher rates of recurrence in the HR/RFA group. The recurrence rates were $42 \%$ in the HR/RFA group and $55 \%$ in the subset of HR/RFA patients with MELD $<10$ and APRI $\leq 0.5$, compared to $8.7 \%$ in the $L T$ group. The disparity between the higher survival despite a higher recurrence rate in the subset of HR/RFA patients with MELD < 10 and APRI $\leq 0.5$ may be partly explained by the timing of recurrences. Most of the recurrences in the HR/RFA group occurred early (within 2 years), while the low MELD and APRI subset tended to have late recurrences (after 2 years). A retrospective study by Portolani et al. ${ }^{[20]}$ which examined intrahepatic recurrence of $\mathrm{HCC}$ after resection found that survival was significantly better in patients with late recurrence compared to early recurrence: $61.9 \%$ vs. $25.7 \%$ at 3 years, and $27.1 \%$ vs. $4.5 \%$ at 5 years. The authors also found that patients with late recurrences were more likely to be cured by resectional or ablative 
therapy of the tumor recurrence, and had survival comparable to those without recurrence. These differences support our hypothesis that careful patient selection, based on characteristics that predict a low level of hepatic parenchyma fibrosis and preserved synthetic function, can identify patients who will have a good long-term outcome after non-transplant therapies.

Although APRI is not widely used in liver transplant literature, we propose that this can be a helpful tool. Liver function can be inferred by prognostic scores such as CTP, MELD or functional tests such as Indocyanine Green. Degree of fibrosis can be assessed more directly by measuring hepatic vein pressures, liver biopsy or transient elastography. These tests are often limited by operator-dependence, biopsy interpretation, sample error, body habitus, and invasiveness. Prognostic scores have been predictive of short-term outcome and survival on a transplant list but these scores were not used specifically to assess fibrosis, longer-term prognosis or predisposition for recurrent cancer. APRI is easy to calculate at the bedside with readily available laboratory parameters and does not require an expensive or invasive test. We found that while an APRI $\leq 0.5$ was correlated with a statistically significant OR for both 3-year and 5-year OS in the HR, RFA, and composite HR/RFA groups, an $A P R I \leq 1.00$ did not predict a survival advantage. $A P R I$ is a reasonable surrogate for fibrosis and our study has shown that when used with MELD $<10$, this has prognostic significance.

This study is limited by its retrospective nature and relatively small sample size. Due to the small sample size, we were unable to report on the outcomes following other non-transplant treatments such as TACE and Yttrium-90. It will be necessary to validate these results in a larger prospective study. This analysis also reported only overall survival, as some patients had recurrence of HCC that was treated but died of liver failure or an unrelated problem. It is thus difficult to determine the exact effect of HCC on survival. Because it was a retrospective study, we did not account for patient comorbidities that may have affected candidacy for transplant or overall survival. This is evident by the older age of the patients who underwent RFA who were not likely to be transplant candidates because of comorbidities.

Despite these limitations, the strength of this study is that this represents a single center experience in which patients are referred to a single group of surgeons who perform most of the liver resections and all of the transplants in the state. The surgeons, hepatologists, oncologists and interventional radiologists are closely associated, so multidisciplinary management allowed equal access to all treatment modalities. Finally, this study was conducted in a small state with a high burden of HCC and has long term follow-up of both transplant and non-transplant patients.

In conclusion, this study suggests that patients with single $\mathrm{HCC}$ tumors $\leq 3 \mathrm{~cm}$, with an APRI $\leq 0.5$ and MELD score < 10 , have an OS after resection or ablation similar to patients undergoing transplantation. Recurrences are higher in this group than patients who underwent transplantation, however recurrences tended to occur late ( $>2$ years). While liver transplantation remains the optimal treatment for $\mathrm{HCC}$, perhaps this subset of patients can safely wait until a more urgent reason for transplant arises, in areas where donor livers are limited. Future studies validating this in a larger population could assist in directing patients with good prognosis to nontransplant therapies, and allow allocation of scarce donor livers to patients with a greater need.

\section{Authors' contributions}

Concept/design, data acquisition, critical revision: L.L. Wong

Manuscript preparation, data analysis: J. Sarkar

Data analysis: T. DeLeon

\section{Financial support and sponsorship}

This paper was supported by NIH2 P30 CA07178913.

\section{Conflicts of interest}

There are no conflicts of interest.

\section{Patient consent}

As this was a retrospective study, the IRB did not require patient consent.

\section{Ethics approval}

This study received IRB approval at the University of Hawaii.

\section{REFERENCES}

1. Mittal S, El-Serag HB. Epidemiology of HCC: consider the population. J Clin Gastroenterol 2013;47:S2-6.

2. Ferlay J, Soerjomataram I, Dikshit R, Eser S, Mathers C, Rebelo M, Parkin DM, Forman D, Bray F. Cancer incidence and mortality worldwide: sources, methods and major patterns in GLOBOCAN 2012. Int J Cancer 2015;136:E359-86.

3. Belghiti J. Transplantation for liver tumors. Semin Oncol 2005;32:2932 . 
4. Yao FY, Bass NM, Nikolai B, Davern TJ, Kerlan R, Wu V, Ascher NL, Roberts JP. Liver transplantation for hepatocellular carcinoma: analysis of survival according to the intention-to-treat principle and dropout from the waiting list. Liver Transpl 2002;8:873-83.

5. Adam R, Bhangui P, Vibert E, Azoulay D, Pelletier G, Duclos-Vallée JC, Samuel D, Guettier C, Castaing D. Resection or transplantation for early hepatocellular carcinoma in a cirrhotic liver: does size define the best oncological strategy? Ann Surg 2012;256:883-91.

6. Ogihara M, Wong LL, Machi J. Radiofrequency ablation versus surgical resection for single nodule hepatocellular carcinoma: longterm outcomes. HPB (Oxford) 2005;7:214-21.

7. Ueno S, Sakoda M, Kubo F, Hiwatashi K, Tateno T, Baba Y, Hasegawa S, Tsubouchi H; Kagoshima Liver Cancer Study Group. Surgical resection versus radiofrequency ablation for small hepatocellular carcinomas within the Milan criteria. $J$ Hepatobiliary Pancreat Surg 2009;16:359-66.

8. Lu XY, Xi T, Lau WY, Dong H, Xian ZH, Yu H, Zhu Z, Shen F, Wu MC, Cong WM. Pathobiological features of small hepatocellular carcinoma: correlation between tumor size and biological behavior. $J$ Cancer Res Clin Oncol 2011;137:567-75.

9. Wai CT, Greenson JK, Fontana RJ, Kalbfleisch JD, Marrero JA, Conjeevaram HS, Lok AS. A simple noninvasive index can predict both significant fibrosis and cirrhosis in patients with chronic hepatitis C. Hepatology 2003;38:518-26.

10. Farinati F, Sergio A, Baldan A, Giacomin A, Di Nolfo MA, Del Poggio P, Benvegnu L, Rapaccini G, Zoli M, Borzio F, Giannini EG, Caturelli E, Trevisani F. Early and very early hepatocellular carcinoma: when and how much do staging and choice of treatment really matter? A multi-center study. BMC Cancer 2009;9:33.

11. Hsueh KC, Lee TY, Kor CT, Chen TM, Chang TM, Yang SF, Hsieh CB. The role of liver transplantation or resection for patients with early hepatocellular carcinoma. Tumour Biol 2016;37:4193-201.

12. Zhou Z, Lei J, Li B, Yan L, Wang W, Wei Y, Cheng K. Liver resection and radiofrequency ablation of very early hepatocellular carcinoma cases (single nodule $<2 \mathrm{~cm}$ ): a single-center study. Eur $J$ Gastroenterol Hepatol 2014;26:339-44.
13. Chapman WC, Klintmalm G, Hemming A, Vachharajani N, Majella Doyle MB, DeMatteo R, Zaydfudim V, Chung H, Cavaness K, Goldstein R, Zendajas I, Melstrom LG, Nagorney D, JarnaginW. Surgical treatment of hepatocellular carcinoma in North America: can hepatic resection still be justified? J Am Coll Surg 2015;220:62837.

14. Seshadri RM, Besur S, Niemeyer DJ, Templin M, McKillop IH Swan RZ, Martinie JB, Russo MW, Iannitti DA. Survival analysis of patients with stage I and II hepatocellular carcinoma after a liver transplantation or liver resection. HPB (Oxford) 2014;16:1102-9.

15. Bellavance EC, Lumpkins KM, Mentha G, Marques HP, Capussotti L, Pulitano C, Majno P, Mira P, Rubbia-Brandt L, Ferrero A, Aldrighetti L, Cunningham S, Russolillo N, Philosophe B, Barroso E, Pawlik TM. Surgical management of early-stage hepatocellular carcinoma: resection or transplantation? J Gastrointest Surg 2008;12:1699-708.

16. Zheng Z, Liang W, Milgrom DP, Zheng Z, Schroder PM, Kong NS, Yang C, Guo Z, He X. Liver transplantation versus liver resection in the treatment of hepatocellular carcinoma: a meta-analysis of observational studies. Transplantation 2014;27:227-34.

17. Liu PH, Hsu CY, Hsia CY, Lee YH, Huang YH, Chiou YY, Lin HC, Huo TI. Surgical resection versus radiofrequency ablation for single hepatocellular carcinoma $\leq 2 \mathrm{~cm}$ in a propensity score model. Ann Surg 2016;263:538-45.

18. Mehta N, Sarkar M, Dodge JL, Fidelman N, Roberts JP and Yao FY. Intention to treat outcome of T1 hepatocellular carcinoma with the "Wait and Not Ablate" approach until meeting the T2 criteria for liver transplant listing. Liver Transplant 2016;22:178-87.

19. Wong RJ, Wantuck J, Valenzuela A, Ahmed A, Bonham C, Gallo A, Melcher ML, Lutchman G, Concepcion W, Esquivel C, Garcia G, Daugherty T, Nguyen MH. Primary surgical resection versus liver transplantation for transplant-eligible hepatocellular carcinoma patients. Dig Dis Sci 2014;59:183-91.

20. Portolani N, Coniglio A, Ghidoni S, Giovanelli M, Benetti A, Tiberio G, Giulini SM. Early and late recurrence after liver resection for hepatocellular carcinoma: prognostic and therapeutic implications. Ann Surg 2006;243:229-35 